\title{
Differentiating electrophysiological response to decrease and increase negative emotion regulation
}

\author{
WU HaiYan ${ }^{1}$, TANG Ping ${ }^{2}$, HUANG XuShu ${ }^{1}$, HU XiaoQing $^{3} \&$ LUO YueJia ${ }^{1,2}$ * \\ ${ }^{1}$ State Key Laboratory of Cognitive Neuroscience and Learning, Beijing Normal University, Beijing 100875, China; \\ ${ }^{2}$ Sichuan Research Center of Applied Psychology, Chengdu Medical College, Chengdu 610500, China; \\ ${ }^{3}$ Department of Psychology, Northwestern University, Evanston, IL 602208, USA
}

Received May 21, 2012; accepted September 28, 2012

\begin{abstract}
We investigated the reappraisal and the time course of negative emotion regulation by performing event-related potential (ERP) recordings. We found that negative pictures elicited more positive P2 and late positivity potential (LPP) deflections than neutral pictures. This effect occurred between 150-2000 ms post-stimulus. Compared to the emotion maintaining condition, the emotion enhancing condition was associated with higher arousal ratings and displayed increased P2 and LPP amplitudes. The decrease condition was also associated with reduced picture-induced arousal; however, it led to increased P2 and LPP amplitudes. Furthermore, when compared with the maintain condition, both the enhancing and decrease conditions significantly enhanced LPP in the early stage (350-750 ms). Compared to previous studies using western subjects, the negative emotion LPP effects of the present study were shorter in duration and the decrease-emotion condition elicited larger LPPs.
\end{abstract}

emotion regulation, cognitive reappraisal, ERP, P2, LPP

Citation: Wu H Y, Tang P, Huang X S, et al. Differentiating electrophysiological response to decrease and increase negative emotion regulation. Chin Sci Bull, 2013, 58: 1543-1550, doi: 10.1007/s11434-013-5746-x

Emotion regulation refers to the effect of cognitive control over emotional responses, where people modulate their emotional states intentionally and consequently change their emotion process or behavior [1-3]. Many studies have shown that emotion regulation is of great significance in promoting memory retrieval [4], decision making [5], social adjustment [6] and mental health [7,8]. Cognitive reappraisal, as described by Gross, is the most frequently studied emotion regulation strategy that involves modulating emotion through re-construction of emotional settings or stimulus meaning [7,9-12]. Previous behavioral studies showed that cognitive reappraisal, as an effective means of regulation, can weaken feelings and expressions of negative emotions without raising one's physiological arousal level [13]. The cognitive reappraisal method can be sub-divided into three categories when using pictures for experiment stimuli: (1) self-focus cognitive reappraisal, (2)

*Corresponding author (email: Luoyj@bnu.edu.cn) situation-focus cognitive reappraisal, and (3) reality checking $[14,15]$. Researchers have examined the effectiveness of various strategies of emotion regulation and how emotion regulation affects cognition, social behavior, and human well-being [16-19]. In the last decade, many studies have used functional magnetic resonance imaging (fMRI) methods to explore the neuronal basis of emotion regulation for negative emotion. Intriguingly, these studies have revealed the meaningful impact of cognitive control on emotion-related neural responses, such as a decreased amygdala activation [20-25]. Traditionally, the most widely used experimental paradigm in these fMRI studies has involved asking subjects to regulate emotion voluntarily, according to specific instructions [26,27]. In recent years, some researchers have employed ERP methodology to explore the time dynamics that occur in emotion regulation studies [28-32]. For example, Moser and Hajcak examined the temporal course of different cognitive reappraisal by using ERP recordings [5]. They found that when subjects 
were instructed to regulate emotion by using a shifting attention strategy, decrease-regulation (i.e. down-regulation) decreased the LPP amplitude elicited by emotional pictures to a level that was similar to LPP amplitudes when passively viewing neutral pictures. This effect occurred early, taking place only $250 \mathrm{~ms}$ after stimulus onset if the modulation strategy was not limited. Nevertheless, the precise time of the decrease effect varied across different experimental designs. For instance, Moser et al. [3] found that the cognitive reappraisal effect began $400 \mathrm{~ms}$ after stimulus onset when a trial-by-trial study design was used. In contrast, when a block design was used, this effect began only $325 \mathrm{~ms}$ after stimulus onset [33]. Meanwhile, the emotion decrease effect occurred at approximately $200 \mathrm{~ms}$ post-stimulus if the subjects viewed the pictures after cognitive reappraisal exertion [32]. Conversely, upregulation has been shown to increase LPP amplitude [3], even though some studies have observed a ceiling effect related to emotional arousal [5,33]. Moser et al. [3] considered the increase-regulation (i.e., up-regulation) effect to be mediated by a different mechanism than decrease-regulation (i.e., suppression), describing it as occurring only when longer picture presentation duration and a trial by trial design are used.

Despite the recent growth of interest in this area, there is still much to be elucidated. For example, although studies have demonstrated that high-arousal stimuli evoke larger P2 deflections than low-arousal stimuli [33-35], few studies have examined the effect of P2-related emotion regulation. Furthermore, the effect of emotion regulation on LPP amplitude is inconsistent across studies [3,28,32,36]. One possible reason for this lack of stability is the differing baseline conditions used in these studies. For example, some researchers utilized a passive view condition, while others employed an attending condition $[3,28,36]$. Under the passive view condition, subjects were likely to engage in attention distraction, and therefore, the baseline condition may involve a lower workload relative to the up-regulation or down-regulation condition. Moreover, previous studies have not restricted the cognitive reappraisal strategy, nor have they reported which strategies subjects use [28-30]. For instance, an emotion regulation study simply provided subjects an up arrow (" $\uparrow$ ”) or a down arrow (" $\downarrow$ ”) and directed subjects to reduce or increase their negative/positive emotions with their own strategy of choice [37]. Hence, it remains to be demonstrated if the application and process of any particular cognitive reappraisal strategy generates emotion regulation and alters emotional responses.

As mentioned above, the central challenge of the present study is assessing the effect of using a cognitive reappraisal strategy on emotional response. We must train subjects to use different regulation strategies and then require them to only use the cognitive reappraisal strategy. Thus, the first aim of the present study is examine the temporal characteristics of brain ERPs to define at which stage each strategy influences the processing of a negative stimulus in a common emotional response task. Another unique aspect of our experimental design was that we included a maintain emotional response condition to enable us to more intuitively compare the various regulation conditions: increase-regulation, decrease-regulation, and maintain-regulation. Finally, considering that numerous studies demonstrated gender differences in emotional processing or emotion regulation [27,38-42], only female subjects were included to avoid such gender-related effects.

\section{Materials and methods}

\subsection{Materials}

The stimulus set comprised 70 unpleasant (mean valance $=$ 2.82 ; mean arousal $=3.08$ ) and 35 neutral, low arousing color images (mean valence $=5.05$; mean arousal $=0.77$ ) taken mainly from the International Affective Picture System (IAPS). We also took images from the Chinese Affective Picture System (CAPS) to obtain a sufficient amount of material. Unpleasant and neutral images differed significantly from each other on IAPS normative valence and arousal ratings. There were 12 negative and 6 neutral pictures used in the emotional regulation training phase, and 10 negative and 5 neutral pictures used in the practice phase. For formal experiments, 48 negative ( 38 from IAPS and 10 from CAPS) and 24 neutral pictures (20 from IAPS and 4 from CAPS) were used. Similar to the IAPS, CAPS was developed based on the dimensions of emotion; therefore, our results cannot be attributed to the material differences used in our regulation condition. The negative picture set included three general categories of images: poor or injured people, animals, and dirty or disaster-based scenes. The neutral picture set included images of general landscapes, people and daily necessities. All pictures were presented on a 16 -inch screen, and the viewing angle was $18.1 \times 14.2^{\circ}$.

\subsection{Sample collection}

Twenty-one healthy, right-handed, female, Chinese students (mean age: 21.2; age range: 19-25) took part in this study. None of these subjects had a history of neurological or psychiatric disorders. Two participants were excluded as a result of data collection malfunction. Study participation lasted approximately $1 \mathrm{~h}$, and participants were paid $40 \mathrm{Yuan} / \mathrm{h}$.

\subsection{Experiment procedure}

After participants received a general description of the experiment, EEG/EOG sensor electrodes were attached. Participants were then seated in front of a computer monitor (16 inch) approximately $0.5 \mathrm{~m}$ away and given detailed task instructions. Before the formal experiment, subjects completed training for emotion regulation that emphasized sub- 
jects should use the cognitive reappraisal strategy and NOT any other working strategy. In each block, participants first saw a cue word indicating their subsequent task during the upcoming block. The cue word "MAINTAIN", "DECREASE" or "INCREASE" was presented in the center of the screen in white font for $4 \mathrm{~s}$, and participants were instructed to view and respond naturally to the upcoming picture, decrease their emotional response to the picture or increase their emotional response to the picture, respectively. On each trial, a fixation mark (+) was presented for $500 \mathrm{~ms}$ at the beginning and followed by a blank black screen for 400-600 ms. Then, the regulated pictures appeared for 3000 $\mathrm{ms}$, during which time the subject engaged in emotional regulation. An emotion arousal rating screen was presented after the offset of the picture for $3500 \mathrm{~ms}$ and then followed by an evaluation response. The interval between the offset of the evaluation and the following fixation cross was 3000 ms (Figure 1).

In the first block of the task, participants viewed 24 negative and 24 neutral pictures randomly and were instructed to simply view the pictures and maintain a natural emotional feeling (hereafter referred as the maintain condition). This condition was designed to establish a baseline for the effects of the regulation instructions. The second block was the DECREASE block, which asked subjects to decrease their emotional response to 48 negative pictures (hereafter referred to as the decrease condition). The third block was a maintain condition containing 24 negative and 24 neutral pictures. The fourth block was an INCREASE block in which subjects were asked to enhance their emotional re- sponses to 48 negative pictures (hereafter referred to as the increase condition). The order of the two emotion modulation blocks (decrease and increase condition) was counterbalanced across subjects. Instructions for the decrease and increase conditions were precisely adapted from Moser et al. [5]. However, instructions for the maintenance condition asked subjects to focus their attention on the presented picture and hold onto their emotional response until the stimulus disappeared. Subjects were given a post-experiment questionnaire after the formal ER procedure to examine the application and effectiveness of the various modulation strategies.

\subsection{Data collection and analysis}

EEG recordings were acquired with the Scan 4.3 software (NeuroScan. Inc., Herndon, Virginia, USA) and obtained from 64 scalp locations, including all standard sites of the International 10/20 system. The EEG was collected with a sample rate of $500 \mathrm{~Hz}$ and a low-pass of $70 \mathrm{~Hz}$. Impedances for all electrodes were kept below $5 \mathrm{k} \Omega$, and all activity was referenced to the right mastoids during the recording. The data were epoched into four categories (Neutral, Maintain-negative, Decrease-negative, Increase-negative) offline, with a pre-stimulus window of -300 to $3000 \mathrm{~ms}$ and band-pass filtered (Butterworth) between 0.5 and $30 \mathrm{~Hz}$ (24 $\mathrm{dB} /$ oct). For each ERP average, the average activity in the $-300-0 \mathrm{~ms}$ window prior to picture onset served as the baseline. Trials in which the absolute amplitude of the signal exceeded $100 \mu \mathrm{V}$ were excluded. The available trial

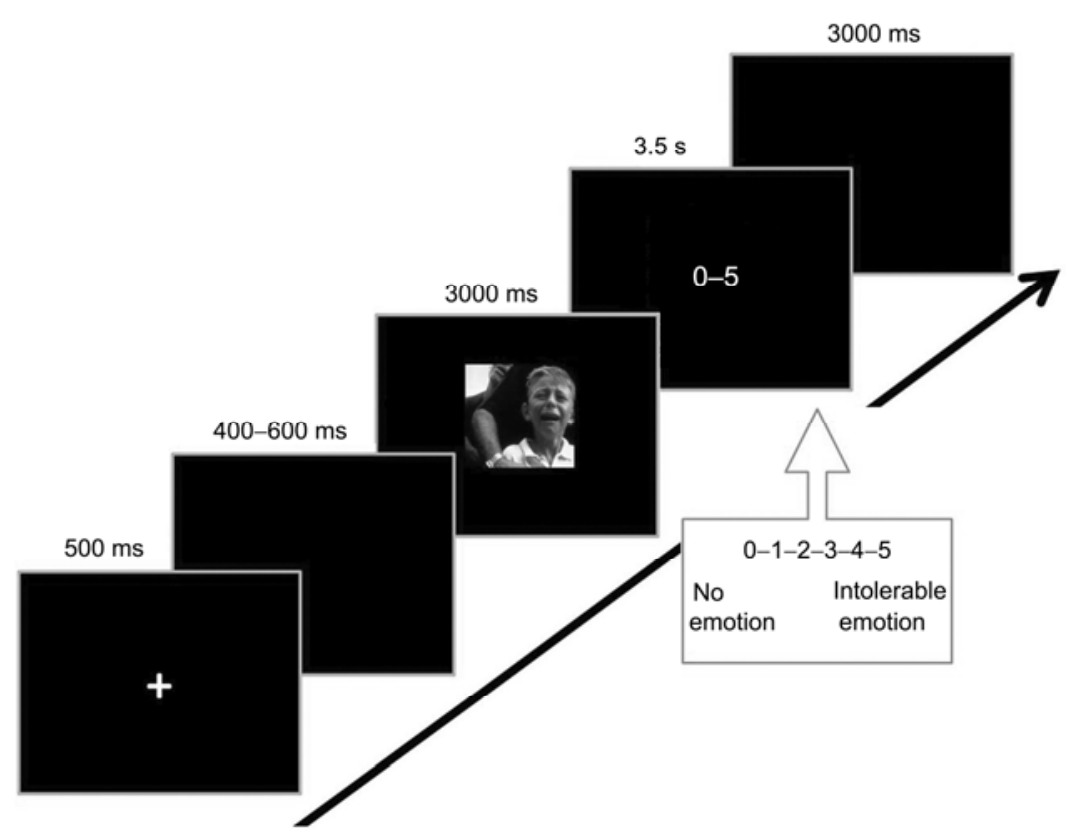

Figure 1 Sample stimuli and procedure for study and test phase. Subjects were presented with a fixation mark (+) was presented for 500 ms at the beginning and followed by a blank black screen for 400-600 ms. Then, the regulated pictures appeared for $3000 \mathrm{~ms}$, during which time the subject engaged in emotional regulation. An emotion arousal rating screen was presented after the offset of the picture for $3500 \mathrm{~ms}$ and then followed by an evaluation response. The interval between the offset of the evaluation and the following fixation cross was $3000 \mathrm{~ms}$. 
numbers in these four conditions were not significantly different, $F(3,54)=0.370, P=0.776$. The mean trial numbers of the four conditions were 46.11, 45.42, 45.37 and 45.74, respectively.

In the present study, nine electrodes $(\mathrm{F} 3, \mathrm{Fz}, \mathrm{F} 4, \mathrm{C} 3, \mathrm{Cz}$, $\mathrm{C} 4, \mathrm{P} 3, \mathrm{Pz}, \mathrm{P} 4)$ were selected to evaluate the ERP components for the P2 and LPP. The P2 was quantified from the eight sites of its baseline-peak amplitude value across the time window of 150-250 ms. However, the LPP amplitude was calculated using six different time windows (TW1: 350-750 ms; TW2: 750-1150 ms; TW3: $1150-1550 \mathrm{~ms}$; TW4: 1550-2000 ms; TW5: 2000-2500 ms; TW6: $2500-3000 \mathrm{~ms}$ ) between the baseline and the average activity in a 500-650 ms window that followed stimulus onset. The data were statistically evaluated using SPSS (Version 13.0) software, and the Analysis of Variance (ANOVA) results were corrected using the Greenhouse-Geisser procedure whenever the sphericity assumption was violated.

\section{Results}

Questionnaire results showed that probability of success for 19 subjects when using cognitive reappraisal during the decrease regulation condition was $70 \%$ to $100 \%(M=$ $86.12 \%)$. For the increase regulation condition, the probability of success ranged from $70 \%$ to $98 \%(M=86.76 \%)$. Moreover, under the decrease condition, the most frequently used strategy was "reality checking" $(M=0.45 \pm 0.24)$, the situation-focus reappraisal method was used less often $(M=$ $0.32 \pm 0.23)$, and the self-focus reappraisal method was used the least $(M=0.19 \pm 0.16)$. The usage preferences of the three reappraisal strategies was significantly different, $F(2,54)=6.82, P<0.01$. The Bonferroni post-test showed the frequency of reality checking was significantly higher than the self-focused reappraisal $(M D=0.26, P<0.01)$. However, in the increase condition, the most frequently used strategy was the situation-focus reappraisal $(M=0.45 \pm$ $0.29)$, reality checking was next $(M=0.28 \pm 0.18)$, and self-focus reappraisal was used the least $(M=0.23 \pm 0.21)$. The strategy preferences of the three reappraisal methods were also significantly different, $F(2,54)=4.90, P<0.05$. Further analysis showed that usage of the situation-focus reappraisal was significantly higher than usage of the self-focus reappraisal $(M D=0.22, P<0.05)$. Because we placed strict limitations on which strategies to use, the probability that subjects used another strategy in addition to these three methods is extremely low (decrease condition: $M=0.07 \pm 0.23$; increase condition: $M=0.04 \pm 0.10$ ), implying we successfully manipulated their usage of the cognitive reappraisal strategy.

Furthermore, the effectiveness ratings of the three reappraisal strategies revealed an almost significant main effect for strategy type in the decrease condition, $F(2,45)=3.19$, $P=0.051$. Reality checking was most effective $(M=4.84 \pm$ $0.38)$, while situation-focus reappraisal was the worst $(M=$
$4.26 \pm 0.81)$. Further tests revealed significant differences between the two $(M D=0.58, P<0.05)$. For increase emotion regulation, there were no significant differences found among the three types of cognitive reappraisal methods.

ANOVA analysis of self-reported emotion ratings repeated with four levels showed a significant emotional arousal difference in four experimental conditions $3000 \mathrm{~ms}$ after picture onset, $F(3,16)=129.49, P<0.001$. The arousal rating under these four condition (Neutral: $0.72 \pm 0.46$, Maintain-negative: $1.55 \pm 0.96)$, Negative-decrease $(M=$ $3.06 \pm 0.83)$, and Increase-negative $(M=3.93 \pm 0.64)]$ were significantly different from each other, $P<0.01$. More specifically, the arousal evaluation of the Maintainnegative condition was significantly higher than the arousal of the Decrease-negative condition and lower than the Increase-negative condition. As shown above, the decreases and increases in emotion regulation changed the subjective emotional experience.

Figure 2 shows the grand averaged ERP waveforms at Fz, $\mathrm{Cz}$ and $\mathrm{Pz}$ Grand average waveform showed the largest $\mathrm{P} 2$ was over the posterior sites (P3, Pz, P4. $M=7.6 \pm 1.1 \mu \mathrm{V}$ ) with smallest amplitude over frontal sites $(M=2.8 \pm 1.5 \mu \mathrm{V})$. LPP appeared from $320 \mathrm{~ms}$ and lasted approximately 2000 ms after pictures onset, while the LPP lasted until the picture disappeared under the increase condition.

To explore the emotional effect in the maintain condition, a 2 maintain (Maintain-negative, Neutral) $\times 9$ electrode (F3, $\mathrm{Fz}, \mathrm{F} 4, \mathrm{C} 3, \mathrm{Cz}, \mathrm{C} 4, \mathrm{P} 3, \mathrm{Pz}, \mathrm{P} 4)$ repeated measurement ANOVA on the $\mathrm{P} 2$ amplitude revealed a significant main effect for the emotion type, $F(1,18)=16.64, P<0.001$, $\eta_{p}{ }^{2}=0.48$, indicated by a P2 value for the Maintain-negative condition $(M=4.7 \mu \mathrm{V})$ that is more positive than the Neutral condition $(M=2.9 \mu \mathrm{V})$. The emotional effect under the maintain condition was also observed in LPP amplitude values for TW1 $\left(F(1,18)=9.34, P<0.01, \eta_{p}{ }^{2}=0.34\right)$, TW2 $\left(F(1,18)=98.13, P<0.0001, \eta_{p}{ }^{2}=0.85\right)$, TW3 $(F(1,18)=$ 38.32, $\left.P<0.0001, \eta_{p}{ }^{2}=0.68\right)$, TW4: $(F(1,18)=18.66, P<$ $\left.0.001, \eta_{p}{ }^{2}=0.51\right)$. These results reveal that Maintainnegative condition elicited a significantly more positive LPP than the Neutral condition did from 350-2000 ms.

As Figure 2 and Table 1 show, there is a consistent trend under the three negative picture conditions for increaseregulation to elicit the most positive LPP amplitude, whereas the Maintain-negative condition evoked minimal LPP amplitude.

A repeated measurement ANOVA of $\mathrm{P} 2$ for the 3 regulation conditions (Maintain-negative; Decrease-negative; Increase-Negative) $\times 9$ electrode $(\mathrm{F} 3, \mathrm{Fz}, \mathrm{F} 4, \mathrm{C} 3, \mathrm{Cz}, \mathrm{C} 4, \mathrm{P} 3$, $\mathrm{Pz}, \mathrm{P} 4)$ showed the main effect of the regulation condition $\left(F(2,17)=5.02, P<0.05, \eta_{p}^{2}=0.22\right)$, indicating the Increase-negative condition $(M=5.9 \pm 1.2 \mu \mathrm{V})$ evoked a more positive $\mathrm{P} 2$ than the Maintain-negative condition $(M=$ $4.7 \pm 1.3 \mu \mathrm{V}), P<0.05$. However, the difference between the Maintain-negative condition and the Decrease-negative condition did not reach statistical significance, $P=0.77$. A 

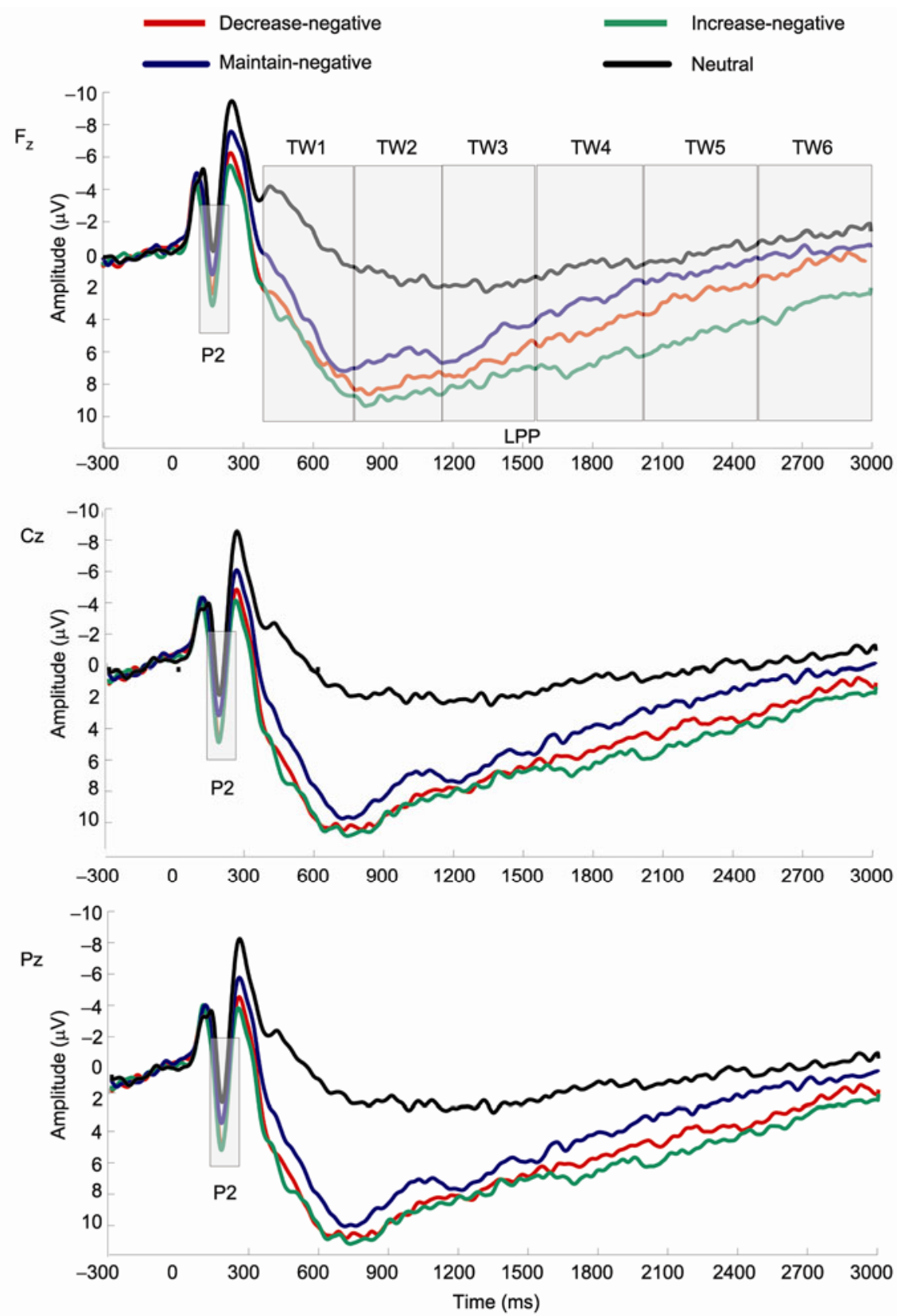

Figure 2 Grand average ERPs elicited by 4 conditions. Data were depicted at 3 representative scalp electrodes: Fz, Cz, Pz. Amplitudes were displayed in $\mu \mathrm{V}$.

Table 1 Mean (SD) P2 and LPP magnitudes (in Microvolts) for 4 conditions

\begin{tabular}{|c|c|c|c|c|}
\hline & Neutral & Maintain-negative & Decrease-negative & Increase-negative \\
\hline $\mathrm{P} 2$ & $2.92(1.22)$ & $4.71(1.25)$ & $5.78(1.34)$ & $5.92(1.22)$ \\
\hline LPP(350-750 ms) & $0.14(0.88)$ & $5.86(1.10)$ & $7.36(1.26)$ & $7.47(1.00)$ \\
\hline LPP(1150-1550 ms) & $1.30(0.99)$ & $4.71(0.86)$ & $5.88(1.20)$ & $6.15(0.78)$ \\
\hline LPP(1550-2000 ms) & $0.10(0.95)$ & $2.64(0.85)$ & $4.32(1.33)$ & $5.12(0.76)$ \\
\hline LPP(2000-2500 ms) & $-0.98(0.92)$ & $0.42(1.00)$ & $2.10(1.45)$ & $3.21(0.84)$ \\
\hline $\operatorname{LPP}(2500-3000 \mathrm{~ms})$ & $-1.98(0.99)$ & $-0.95(1.03)$ & $0.20(1.55)$ & $1.36(0.83)$ \\
\hline
\end{tabular}


significant main effect of the electrode, $F(8,11)=14.02, P$ $<0.001, \eta_{p}{ }^{2}=0.44$, showed that the P2 at F3, F4, Fz and C3 was smaller than the $\mathrm{P} 2$ for $\mathrm{Cz}, \mathrm{P} 3, \mathrm{P} 4$ and Pz.

For LPP amplitude occurring at 350-3000 ms, the repeated measurement ANOVA for 3 regulation conditions (Maintain-negative, Decrease-negative and Increase-negative $) \times 9$ electrode $(\mathrm{F} 3, \mathrm{Fz}, \mathrm{F} 4, \mathrm{C} 3, \mathrm{Cz}, \mathrm{C} 4, \mathrm{P} 3, \mathrm{Pz}, \mathrm{P} 4)$ only showed a significant effect of the regulation condition for TW1, $F(2,17)=5.91, P<0.01, \eta_{p}{ }^{2}=0.25$. Further analysis revealed that the Maintain-negative condition $(M=5.9 \pm$ $1.1 \mu \mathrm{V})$, elicited significantly smaller LPP amplitude than the increase $(M=7.5 \pm 1.0 \mu \mathrm{V})$ and decrease conditions $(M$ $=7.4 \pm 1.3 \mu \mathrm{V})$. The significant interaction of condition $\times$ electrode in some LPP time windows indicates that the increase-regulation condition evoked more positive potentials than maintain condition from P2 to LPP (TW1 to TW6) in most electrodes, while the difference between the decrease regulation condition and the maintain condition was significant only for the P2(C3, C4) and LPP in TW1 (F3, Fz, F4, $\mathrm{C} 3, \mathrm{Cz}, \mathrm{C} 4)$.

\section{Discussion}

In this study, we examined the effects of instructing subjects to intentionally modulate their emotional responses to electrophysiological activity elicited by viewing negative-valence emotion pictures. Our behavioral results and the regulation strategies used partially replicate the findings of previous studies. One notable difference was most of our subjects reported using reality checking in a negative-decrease task, while previous research using western subjects showed the dominance of the self-focus strategy [33]. Many studies show that culture shapes our self-concept, and this difference may be partially attributed to the self-structure differences between western and eastern individuals [43-48]. As the literature highlights, the western self is independent, while the eastern self is interdependent; eastern individuals may focus on relationships with others and have a higher sensitivity to context $[49,50]$. Therefore, it is possible that eastern culture influences subjects' reappraisal strategy preferences, leading these subjects to utilize the self-focused strategy the least in both the increase and decrease regulation conditions, in contrast to previous western subjects' preference for a self-focus strategy.

We observed significant ERP differences between the Maintain-Negative and Neutral conditions. Specifically, the Maintain-Negative condition elicited a larger P2 and LPP than the Neutral condition. In contrast to prior reports showing that emotion effects continued until the stimuli disappeared, we observed an emotion effect from $150 \mathrm{~ms}$ to $2000 \mathrm{~ms}$ after the pictures were presented [3]. The negative stimulus did evoke a trend toward a more positive LPP 2000-3000 ms after stimulus onset, but the difference did not reach statistical significance. To some extent, our results are consistent with a recent ERP study showing an emotional modulation of the LPP and an emotion effect lasting up to $6 \mathrm{~s}$ in Chinese subjects [51].

Consistent with previous studies [3,26,52], we found that the Increase-Negative condition was associated with more enhanced positive potential than the Maintain-negative condition, and this difference started $150 \mathrm{~ms}$ (i.e., P2) after stimulus onset. These ERP differences indicate a regulation effect and can be interpreted as an increase in attention and arousal processes due to more negative emotions [53,54]. Previous studies demonstrate that the attention effect related to negativity bias was associated with P2 (or P200) [53,55], and this could explain the enhanced P2 in the Increase-negative condition. Additionally, studies on emotional picture processing have suggested that LPPs are higher with high arousal affective pictures than with less affectively intense pictures [56,57]. Intriguingly, we observed two peaks at 350-750 ms and 2000-2500 ms post-stimulus onset, which contrasts with prior research suggesting the emotion regulation effect, is stable throughout the LPPs. However, larger individual differences may account for this unstable effect.

Our findings in the Decrease-negative trials differed from previous research. Rather than observing a decrease in LPP amplitude, we found a slight increase. Interestingly, we found that the regulation effect of decrease instructions only occurs 350-700 ms after stimulus onset (LPP), over the frontal and central electrodes, with Decrease-negative trials eliciting more positive LPPs than Maintain-negative trials. This result may reflect a suppression effect on early LPP (P300).

There are several possible reasons why the electrophysiological response did not differ significantly between the decreases and maintain trials beyond the 350-750 ms time window in our study. First, it is possible that the unrestricted cognitive reappraisal strategy increased variance (individual differences) in the context of the current study. Indeed, the standard deviations of the LPP segments in the decrease condition were larger than those in the other conditions. Furthermore, the reappraisal strategy preference difference between Chinese and western subjects may also produce different LPP patterns. Most of our subjects reported using a reality-checking strategy in the Decrease-negative condition, while western subjects previously reported using a self-focused strategy. Additionally, employing the Maintain-negative as a baseline condition requires subjects to intentionally sustain each emotional response until stimulus offset, which is intended to make the cognitive control component among the decrease, increase, and maintain conditions similar. Moreover, motivation may play an important role because the Decrease-negative condition may be more difficult for Chinese individuals and thereby require more attention resources and motivation, which would correlate with increased LPP amplitude $[2,58]$. 
The variations in the increase-regulation effect over time that we observed during the $3 \mathrm{~s}$ stimulus viewing period, as well as the presence of the decrease-regulation effect on LPP being limited to the $350-750 \mathrm{~ms}$ post-stimulus onset period, suggests that there is an important task effect in the 350-750 ms time window. Given that studies have shown that LPP amplitude increases under the additional task demand of looking at pictures [36,59], we would expect the addition of an emotional regulation task to increase LPP amplitude regardless of the direction of the regulation. Moreover, the baseline maintain condition may involve a greater workload than a passive view condition, and this should minimize the workload difference between the maintain and regulation conditions. Furthermore, considering the induced emotion may affect the followed negative emotion processing [60], the negative-maintain condition may partly affected by the prior emotion regulation block.

Finally, it is worth note that the decrease-regulation and increase-regulation conditions differed in both time course and electrode distribution of effects. These differences may promote our understanding of these two emotion regulation mechanisms. Our observation of the decrease-regulation effect predominantly at the frontal-central electrodes (especially $350-750 \mathrm{~ms}$ ) is consistent with prefrontal cortex activity inhibiting the limbic system responses [11]. Meanwhile, the presence of the increase regulation effect predominantly at the fronto-central and posterior electrodes is consistent with the prefrontal lobe promoting limbic system activity.

This work was supported by the National Basic Research Program of China (2011CB711000), National Natural Science Foundation of China (91132704, 30930031), National Key Technologies Research and Development Program of China (2009BAI77B01), and the Global Research Initiative Program, National Institutes of Health, USA (1R01TW007897).

1 Liu Y, Fu Q F, Fu X L. The interaction between cognition and emotion. Chin Sci Bull, 2009, 54: 4102-4116

2 Hajcak G, Dunning J P, Foti D. Motivated and controlled attention to emotion: Time-course of the late positive potential. Clin Neurophysiol, 2009, 120: 505-510

3 Moser J S, Krompinger J W, Dietz J, et al. Electrophysiological correlates of decreasing and increasing emotional responses to unpleasant pictures. Psychophysiology, 2009, 46: 17-27

4 Thomas S J, Johnstone S J, Gonsalvez C J. Event-related potentials during an emotional stroop task. Int J Psychophysiol, 2007, 63: 221-231

5 Moser J S, Hajcak G, Bukay E, et al. Intentional modulation of emotional responding to unpleasant pictures: An ERP study. Psychophysiology, 2006, 43: 292-296

6 Schwartz D, Proctor L J. Community violence exposure and children's social adjustment in the school peer group: The mediating roles of emotion regulation and social cognition. J Consult Clin Psych, 2000, 68: 670-683

7 Gross J J. Emotion regulation: Affective, cognitive, and social consequences. Psychophysiology, 2002, 39: 281-291

8 Horn A B, Ehrhard K, Traue H C, et al. Neural correlates of emotion regulation and mental health. Psychol Health, 2006, 21: 68

9 Gross J J. Emotion regulation in adulthood: Timing is everything.
Curr Dir Psychol Sci, 2001, 10: 214-219

10 Gross J J, John O P. Individual differences in two emotion regulation processes: Implications for affect, relationships, and well-being. $\mathrm{J}$ Pers Soc Psychol, 2003, 85: 348-362

11 Ochsner K N, Gross J J. The cognitive control of emotion. Trends Cogn Sci, 2005, 9: 242-249

12 Gross J J. Handbook of Emotion Regulation. New York: Guilford Press, 2007

13 Egloff B, Schmukle S C, Burns L R, et al. Spontaneous emotion regulation during evaluated speaking tasks: Associations with negative affect, anxiety expression, memory, and physiological responding. Emotion, 2006, 6: 356-366

14 Abler B, Erk S, Herwig U, et al. Anticipation of aversive stimuli activates extended amygdala in unipolar depression. J Psychiatr Res, 2007, 41: 511-522

15 Herwig U, Baumgartner T, Kaffenberger T, et al. Modulation of anticipatory emotion and perception processing by cognitive control. NeuroImage, 2007, 37: 652-662

16 Bebko G M, Franconeri S L, Ochsner K N, et al. Look before you regulate: Differential perceptual strategies underlying expressive suppression and cognitive reappraisal. Emotion, 2011, 11: 732-742

17 Blechert J, Sheppes G, Di Tella C, et al. See what you think: Reappraisal modulates behavioral and neural responses to social stimuli. Psychol Sci, 2012, 23: 346-353

18 Dan-Glauser E S, Gross J J. The temporal dynamics of two response-focused forms of emotion regulation: Experimential, expressive, and autonomic consequences. Psychophysiology, 2011, 48: 1309-1322

19 Denson T F, Grisham J R, Moulds M L. Cognitive reappraisal increases heart rate variability in response to an anger provocation. Motiv Emotion, 2011, 35: 14-22

20 Winecoff A, Labar K S, Madden D J, et al. Cognitive and neural contributors to emotion regulation in aging. Soc Cogn Affect Neurosci, 2011, 6: 165-176

21 Mcrae K, Hughes B, Chopra S, et al. The neural bases of distraction and reappraisal. J Cogn Neurosci, 2010, 22: 248-262

22 Mcrae K, Misra S, Prasad A K, et al. Bottom-up and top-down emotion generation: Implications for emotion regulation. Soc Cogn Affect Neur, 2012, 7: 253-262

23 Koenigsberg H W, Fan J, Ochsner K N, et al. Neural correlates of using distancing to regulate emotional responses to social situations. Neuropsychologia, 2010, 48: 1813-1822

24 Payer D E, Baicy K, Lieberma N M D, et al. Overlapping neural substrates between intentional and incidental down-regulation of negative emotions. Emotion, 2012, 12: 229-235

25 Ochsner K N, Bunge S A, Gross J J, et al. Rethinking feelings: An fMRI study of the cognitive regulation of emotion. J Cogn Neurosci, 2002, 14: 1215-1229

26 Ochsner K N, Ray R D, Cooper J C, et al. For better or for worse: Neural systems supporting the cognitive down- and up-regulation of negative emotion. NeuroImage, 2004, 23: 483-499

27 Mcrae K, Ochsner K N, Mauss I B, et al. Gender differences in emotion regulation: An fMRI study of cognitive reappraisal. Group Process Interg, 2008, 11: 143-162

28 Moser J S, Most S B, Simons R F. Increasing negative emotions by reappraisal enhances subsequent cognitive control: A combined behavioral and electrophysiological study. Cogn Affect Behav Neurosci, 2010, 10: 195-207

29 Kisley M A, Campbell A M, Larson J M, et al. The impact of verbal framing on brain activity evoked by emotional images. J Integr Neurosci, 2011, 10: 513-524

30 Macnamara A, Ochsner K N, Hajcak G. Previously reappraised: The lasting effect of description type on picture-elicited electrocortical activity. Soc Cogn Affect Neurosci, 2011, 6: 348-358

31 Walker S, O'connor D B, Schaefer A. Brain potentials to emotional pictures are modulated by alexithymia during emotion regulation. Cogn Affect Behav Neurosci, 2011, 11: 463-475

32 Hajcak G, Nieuwenhuis S. Reappraisal modulates the electrocortical response to unpleasant pictures. Cogn Affect Behav Neurosci, 2006, 
6: 291-297

33 Krompinger J W, Moser J S, Simons R F. Modulations of the electrophysiological response to pleasant stimuli by cognitive reappraisal. Emotion, 2008, 8: 132-137

34 Hirata K, Pal I, Lehmann D. Event-related potential components N1, P2 and P3 to rare and frequent stimuli in intellectually impaired neurological patients. Eur Arch Psychiatry Clin Neurosci, 1991, 240: 240-245

35 Yuan J J, Zhang Q L, Chen A T, et al. Are we sensitive to valence differences in emotionally negative stimuli? Electrophysiological evidence from an ERP study. Neuropsychologia, 2007, 45: 2764-2771

36 Hajcak G, Moser J S, Simons R F. Attending to affect: Appraisal strategies modulate the electrocortical response to arousing pictures. Emotion, 2006, 6: 517-522

37 Mak A K Y, Hu Z G, Zhang J X, et al. Neural correlates of regulation of positive and negative emotions: An fMRI study. Neurosci Lett, 2009, 457: 101-106

38 Mcbain R, Norton D, Chen Y E. A female advantage in basic face recognition is absent in schizophrenia. Psychiat Res, 2010, 177: 12-17

39 Bourne V J, Maxwell A M. Examining the sex difference in lateralisation for processing facial emotion: Does biological sex or psychological gender identity matter? Neuropsychologia, 2010, 48: 1289-1294

40 Stevens J S, Hamann S. Sex differences in brain activation to emotional stimuli: A meta-analysis of neuroimaging studies. Neuropsychologia, 2012, 50: 1578-1593

41 Hofmann S G, Suvak M, Litz B T. Sex differences in face recognition and influence of facial affect. Pers Indiv Differ, 2006, 40: 1683-1690

42 Yuan J J, Xu S, Li C Q, et al. The enhanced processing of visual novel events in females: ERP correlates from two modified three-stimulus oddball tasks. Brain Res, 2012, 1437: 77-88

43 Markus H, Kitayama S. Culture and the self: Implications for cognition, emotion, and motivation. Psychol Rev, 1991, 98: 224-253

44 Ishii K, Kobayashi Y, Kitayama S. Interdependence modulates the brain response to word-voice incongruity. Soc Cogn Affect Neurosci, 2010, 5: 307-317

45 Ray R D, Shelton A L, Hollon N G, et al. Interdependent self-construal and neural representations of self and mother. Soc Cogn Affect Neurosci, 2010, 5: 318-323

46 Zhu Y, Zhang L, Fan J, et al. Neural basis of cultural influence on self-representation. NeuroImage, 2007, 34: 1310-1316

47 Oyserman D, Lee S W S. Does culture influence what and how we think? Effects of priming individualism and collectivism. Psychol Bull, 2008, 134: 311-342

48 Chiao J Y, Harada T, Komeda H, et al. Neural basis of individualistic and collectivistic views of self. Hum Brain Mapp, 2009, 30: 2813-2820

49 Doherty M J, Tsuji H, Phillips W A. The context sensitivity of visual size perception varies across cultures. Perception, 2008, 37: 1426-1433

50 Goto S G, Ando Y, Huang C, et al. Cultural differences in the visual processing of meaning: Detecting incongruities between background and foreground objects using the N400. Soc Cogn Affect Neurosci, 2010, 5: 242-253

51 Yen N S, Chen K H, Liu E H. Emotional modulation of the late positive potential (LPP) generalizes to Chinese individuals. Int J Psychophysiol, 2010, 75: 319-325

52 Jackson D C, Malmstadt J R, Larson C L, et al. Suppression and enhancement of emotional responses to unpleasant pictures. Psychophysiology, 2000, 37: 515-522

53 Huang Y X, Luo Y J. Temporal course of emotional negativity bias: An ERP study. Neurosci Lett, 2006, 398: 91-96

54 Olofsson J K, Nordin S, Sequeira H, et al. Affective picture processing: An integrative review of ERP findings. Biol Psychol, 2008, 77: 247-265

55 Carretie L, Mercado F, Tapia M, et al. Emotion, attention, and the 'negativity bias', studied through event-related potentials. Int $\mathbf{J}$ Psychophysiol, 2001, 41: 75-85

56 Schupp H T, Cuthbert B N, Bradley M M, et al. Affective picture processing: The late positive potential is modulated by motivational relevance. Psychophysiology, 2000, 37: 257-261

57 Schupp H T, Junghofer M, Weike A I, et al. The selective processing of briefly presented affective pictures: An ERP analysis. Psychophysiology, 2004, 41: 441-449

58 Lee K Y, Lee T H, Yoon S J, et al. Neural correlates of top-down processing in emotion perception: An ERP study of emotional faces in white noise versus noise-alone stimuli. Brain Res, 2010, 1337: 56-63

59 Hajcak G, Dunning J P, Foti D. Neural response to emotional pictures is unaffected by concurrent task difficulty: An event-related potential study. Behav Neurosci, 2007, 121: 1156-1162

60 Chen C P, Luo Y J. Attentional negativity bias moderated by positive mood arousal. Chin Sci Bull, 2010, 55: 2010-2015

Open Access This article is distributed under the terms of the Creative Commons Attribution License which permits any use, distribution, and reproduction in any medium, provided the original author(s) and source are credited. 